\title{
APORTACIONES AL ESTUDIO DE LOS MOVIMIENTOS MIGRATORIOS: LAS REPÚBLICAS DEL CAUCASO
}

\author{
José GARRIDO PALACIOS \\ Departamento de Geografía y Ordenación del Territorio \\ Universidad de Zaragoza
}

Resumen: En este trabajo se estudian los movimientos de refugiados y desplazados internos de las Repúblicas del Cáucaso (Armenia, Azerbaiyán y Georgia) mediante la elaboración de mapas de flujos.

Palabras clave: Refugiados, desplazados internos, Repúblicas del Cáucaso y mapas de flujos.

Abstract: This work studies the refugees and internally displaced persons of the republics of Caucasus (Armenia, Azerbaijan and Georgia) with flow maps.

Key words: Refugees, internally displaced persons, republics of Caucasus and flow maps

\section{INTRODUCCION}

La cartografía comprende "el conjunto de estudios y operaciones científicas, artísticas y técnicas que intervienen a partir de los resultados de las observaciones directas o de la explotación de la documentación, en vistas a la elaboración y a 1 establecimiento de mapas, planos y otras formas de expresión, así como de su utilización" (ASOCIACIÓN CARTOGRÁFICA INTERNACIONAL, 1966). Así, la cartografía es, pues, el instrumento que permite estudiar los problemas territoriales. Es también una fuente de información de primer orden y base para la investigación a l suscitar problemas y contribuir a su conocimiento.

En este sentido, la cartografía nos permitirá observar determinados aspectos de los movimientos migratorios mediante la utilización de las variables visuales ad hoc: tamaño, valor, textura, forma, orientación y color (CALVO, 1992). 
Este trabajo pretende aproximarse al conocimiento de los movimientos de población de las Repúblicas del Cáucaso (Armenia, Azerbaiyán y Georgia), tanto abordando los desplazamientos internos como de refugiados, mediante la presentación corpus de mapas que transmita la idea del problema (PUEYO, 1994), en especial los flujos migratorios durante los años 1992, 1993 y 1994. Este tipo de cartografía expresa sin duda la trascendencia del hecho y permite comparar los desplazamientos espaciales y temporales, si bien el período de estudio es reducido por tratarse de una problemática reciente.

La independencia de estas Repúblicas después de la caída del régimen comunista de la extinta URSS, en diciembre de 1991, ha propiciado movimientos de población a gran escala por dos motivos esenciales: el afán secesionista de regiones específicas (Abjasia y Osetia del Sur) y la anexión de enclaves en Armenia y Azerbaiyán con mayoría étnica distinta de la propia del país.

Ambas causas constituyen el desencadenante de unos conflictos complejos que afectan a millón y medio de migrantes (en torno al $10 \%$ de la población total) en ese ámbito geográfico, donde el aspecto bélico está vinculado con otros de naturaleza económical, social y especialmente étnica ${ }^{2}$.

Ahora bien, hoy más que nunca, los refugiados forman parte de un fenómeno migratorio, en el que factores políticos, étnicos, económicos, medioambientales y de derechos humanos se combinan y conducen a los movimientos de población (ACNUR, 1995).

\section{FUENTES Y METODOLOGIA}

Se carece de homogeneidad estadística en el tratamiento de la información para el estudio de los movimientos migratorios, en particular las personas incluidas en

\footnotetext{
1 ZOLBERG (1991) afirma que en las Repúblicas ex-socialistas el desempleo se ha incrementado, y ello conlleva el éxodo de población de esos países hacia otros de mayor bienestar. En el mismo sentido, WEINTRAUB (1989) postula que los movinientos migratorios reaccionan ante un determinado número de cstimulos, en su mayoría de indole conónica.

2 El conflicto étnico se define como un enfrentamiento violento entre dos o más grupos que difieren en cuanto a su cultura, religión, características físicas o idioma (LEVINSON, 1993). El mismo autor tipifica los conflictos étnicos en cuatro categorías, de modo que en el grupo de "movimientos separatistas" engloba a las Repúblicas caucasianas.

El ACNUR (1994, p. 20) considera asimismo que las tensiones étnicas han proliferado en los ultimos años en Amenia, Azerbaiyán, Georgia,... En suma, parece que la causa principal de las migraciones que se producen en el ámbito de estudio es esencialmente étnico.
} 
el grupo de refugiados ${ }^{3}$. Así pues, existen dos fuentes principales sobre las migraciones internacionales: el Alto Comisionado de las Naciones Unidas (ACNUR o UNHCR en inglés) y el Comité para Refugiados de Estados Unidos (USCR) ${ }^{4}$.

Para este estudio se ha elegido la fuente del Comité Estadounidense para los Refugiados porque se ha tenido acceso a una serie más amplia (años 1992, 1993 y 1994) y, por otra parte, la estimación de la información está más cercana a la realidad (tanto de refugiados como de desplazados en el Cáucaso), lo que conlleva a una mejor comprensión de la magnitud del problema de los desplazados 5 y refugiados.

En definitiva, las categorías utilizadas en el trabajo son estas:

\author{
- Refugiados \\ - Desplazados internos \\ - Retornos
}

\begin{abstract}
${ }^{3}$ El término de refugiado es interpretado de diferente forma según el organismo o investigador que trate el tema. Así, la Convención de la ONU en 1951, define a los refugiados como las personas que han dejado su pais porque tienen un temor bien fundado a sufrir persecución, o porque su seguridad se ve amenazada por hechos que perturban graventente el orden público (ACNUR, 1995, p. 244). Esta interpretación es admitida internacionalmente, si bien, por ejemplo, el Comité Estadounidense para los Refugiados (USCR) incluye sólo a los que necesitan protección y/o ayuda y no pueden o no quieren repatriarse por temor a persecución o violencia en su lingar de origen (USCR, 1994). Por tanto, esta definición señala mayor restricción que la anterior.

Finalmente, ZOLBERG (1989, p. 21), de acuerdo a la Convención de 1951, precisa la definición en este sentido:the political events which in the country of origin led to his departure must be accompanied by persecution or the threat of persecution against himself or at least against a section of the population with he identifies himself. En suma, estas interpretaciones difieren y son más o menos restrictivas. En cualquier caso, todas se apoyan en la Convención de Ginebra celebrada en 1951.

Por otra parte, se define desplazado interno como la persona que migra dentro de su propio país por razones de conflicto humano o relocalización forzada (USCR, 1994). Esta definición presenta mayor homogeneidad y admisión en el ámbito internacional, por tanto, no parece conveniente extenderse más en ella. Igualmente existe unidad de criterio en el término de retorno para aludir a la persona que se encuentran fuera del país y regresa a su lugar de origen.

4 En las cifras estadísticas del ACNUR se distingue entre refugiado y "como refugiado". Este último término alude a las personas que no han sido formalmente reconocidas como refugiados pero se benefician de las actividades de protección y ayuda de la organización. En sus estadísticas se describen como "otros" (ACNUR, 1995).

Por su parte, el informe anual del Comité Estadounidense para los Refugiados (USCR) presenta sólo a los refugiados que necesitan protección y/o ayuda y ante la imposibilidad de repatriarse a causa del miedo constante a sufrir persecución en sus patrias y la ausencia de oportunidades de asentamiento permanente en sus países de asilo o en otro lugar. Esta es precisamente la mayor diferencia respecto a las cifras de ACNUR, lo que infiere mayor restricción al término. Al mismo tiempo, el concepto es más global porque incluye en esa situación a los Palestinos.

El informe de Estados Unidos presenta también un grupo de migrantes de selected population in refugee life situntions que no son admitidos como refugiados pero que reciben ayuda temporal (USCR,1994).

5 Desde la óptica de la distancia, ABELLAN (1991,p. 118) distingue entre los movimientos internos o nacionales y los internacionales. Los primeros en nuestro estudio aluden a los desplazamientos internos por razones forzadas ya expuestas; y los segundos incluyen los refugiados conforme a la fuente del Comité Estadounidense para los Refugiados.
\end{abstract}


La metodología aplicada radica en la presentación de un modelo teórico que explica la interrelación de factores que intervienen en las causas de esta problemática, en la exposición del conflicto a partir del acceso a la independencia de las Repúblicas en 1991 y en la representación cartográfica de los flujos de migrantes, desplazados internos y refugiados, y el valor porcentual de población refugiada respecto al total en cada una de las Repúblicas de referencia. Esta cartografía nos permite comparar precisamente el volumen de movimientos de un ámbito a otro y la evolución temporal de los mismos durante los años 1992, 1993 y 1994.

\section{MODELO TEORICO DE LOS FLUJOS MIGRATORIOS}

No es fácil encontrar una teoría general sobre el tema de las migraciones de refugiados habida cuenta de la complejidad de las causas y de los procesos decisorios que están implícitos en los desplazamientos forzosos. No obstante, a modo de ejemplo de esa interrelación de factores que intervienen en el fenómeno de la migración se expone el modelo teórico de WOOD (1994). No se pretende profundizar en exceso en este $u$ otro modelo ad hoc, sino presentar la complejidad del tema y observar que en las Repúblicas del Cáucaso se interrelacionan esos factores.

Wood agrupa los factores que intervienen en los flujos migratorios de esta forma:

- De inestabilidad política, guerra y persecución

- De crisis económicas y ecológicas

- Etnicos, religiosos y tribales

Todos ellos se fusionan en los conflictos del Cáucaso. En efecto, los cambios de gobiernos, las luchas por el poder, asesinatos, etc. son frecuentes en este ámbito a partir de 1991 (EL ESTADO DEL MUNDO, 1992-1996). En segundo lugar, en estos conflictos subyacen intereses económicos, como el caso de Azerbaiyán con la explotación de los yacimientos off-shore en Chirag y Azeri (Mar Caspio) que ha propiciado vivas tensiones en la región. Finalmente, los factores étnicos son los más evidentes por la ubicación de enclaves en Armenia y Azerbaiyán (Figura 2).

En consecuencia, la interrelación de esos factores conducen al desplazamiento de fuertes contingentes de población, tanto internos como de refugiados. Ahora bien, todo no es tan simple como puede parecer a primera vista, pues los recientes flujos de refugiados constituyen en muchos casos episodios contemporáneos de viejos conflictos,

6 Otro modelo se expone en el texto de KUNZ (1973): The Refugec in flight: kinetic models and forms of displacentent 
marcados también por una fuerte intervención externa. Este es el contexto que puede aplicarse también a las Repúblicas caucásicas (ESCALONA, 1995). Pues bien, en el presente trabajo sólo se han esbozado algunas vías de investigación en aras de su desarrollo posterior en otros estudios.

\section{DESARROLLO DE LOS CONFLICTOS}

La República de Georgia, a partir de su acceso a la independencia en 1991, se enfrenta a dos conflictos secesionistas (Figura 1): en Abjasia y en Osetia del Sur. La primera engloba a 525.000 habitantes, de los cuales el $40 \%$ son georgianos; es decir, cristianos ortodoxos, mientras que los abjasianos son musulmanes, lo que infiere un rasgo diferencial importante per se. Esta región reivindica su independencia de Georgia desde 1992 y ha provocado un desplazamiento interno de 270.000 personas y unos 80.000 refugiados, en especial hacia la Federación Rusa y otros estados de la CEI.

La región de Osetia del Sur intenta escindirse de Georgia y aproximarse a Osetia septentrional. Con ese objetivo inició una guerra en 1989 que ha producido unos 120.000 refugiados y 36.000 personas desplazadas dentro de la propia República.

Respecto a Armenia y Azerbaiyán, ambas mantienen litigios por enclaves situados en territorio foráneo (Figura 1). Así, la región de Nagorno-Karabaj? separada de Armenia por una estrecha franja, está ocupada por 190.000 habitantes, siendo el $75 \%$ armenios que reclaman su unión con la República de su misma etnia. En esta línea, las luchas han sido continuas, tanto en el aspecto político, con la declaración de independencia en septiembre de 1991, como a escala bélica, con la toma de Latchín en 1992 que permitía salir del aislamiento en que estaba confinada la región.

Igualmente puede decirse de la región de Nakitcheván situada entre Armenia, Irán y Turquía, cuya mayoría es azerí, y que ha sufrido sucesivos ataques de combatientes armenios. En suma, este conflicto entre armenios y azeríes ha supuesto in desplazamiento de más de un millón de personas, con el consiguiente desarraigo de población.

\footnotetext{
7 El conflicto de este enclave se sumerge en una historia compleja y trágica que se remonta a la guerra armenio-azerí en 1919 y posterior sovietización en 1923. Aunque las reivindicaciones de autonomía e independencia han sido constantes, la situación se agrava tras la desmembración de la URSS en 1991, lo que conlleva grandes desplazamientos de población entre Armenia y Azerbaiyán. En suma, se constata que el conflicto no es reciente, aunque el punto de partida para el estudio sea precisamente la fecha de 1991. (MEDICOS SIN FRONTERAS, 1995),
} 


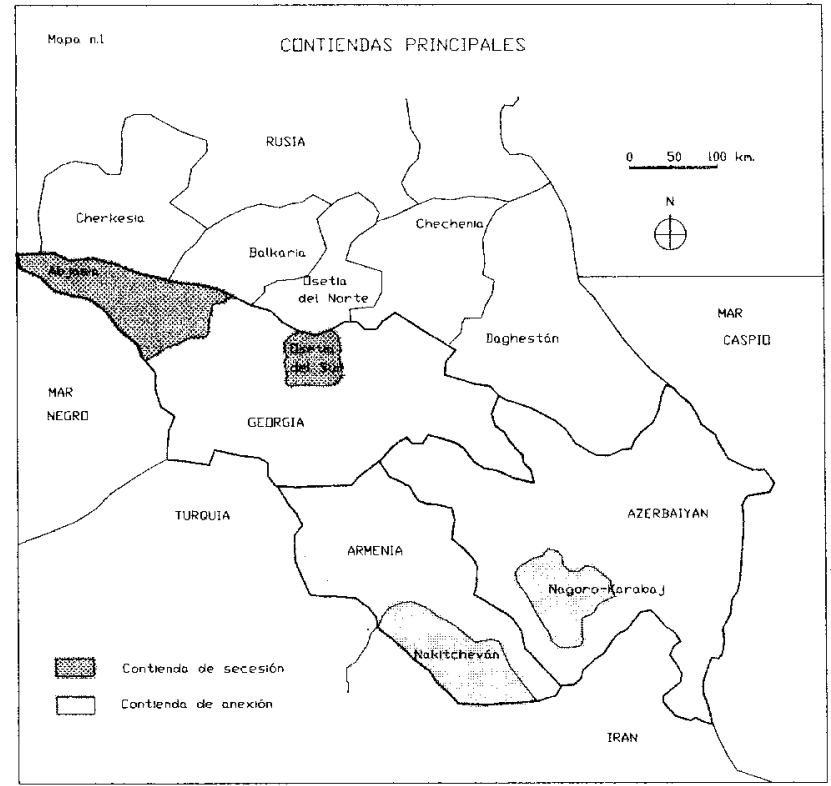

Figura 1.- Contiendas principales.

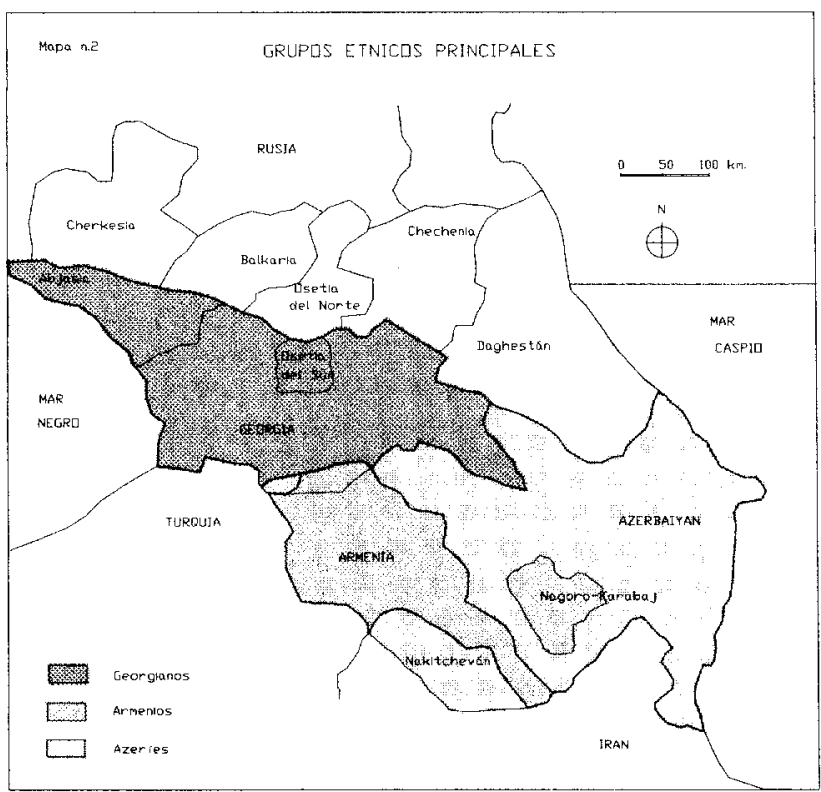

Figura 2.- Principales grupos étnicos. 
Como se ha mentado anteriormente, la raíz de la problemática en este ámbito es de tipo étnico, sin obviar el resto de factores, por lo que la Figura 2 coadyuva a comprender gran parte de la causa principal, en particular en las regiones de NagornoKarabaj y Nakitcheván.

Con todo, la desaparición del régimen comunista y la concomitancia de la lucha por el poder y el territorio han provocado la migración de un elevado número de personas en las Repúblicas caucásicas.

La cartografía nos expresa esos movimientos migratorios, de forma que en primer lugar analizaremos los mapas de flujos durante los años 1992, 1993 y 1994 y posteriormente se verán las características de los desplazados internos.

\section{FLUJOS MIGRATORIOS}

Las migraciones de refugiados más significativas se producen entre las Repúblicas de Armenia y Azerbaiyán, con umbrales comprendidos entre los 200.000 y 300.000 personas durante los años de referencia (Vid cuadro ${ }^{\circ} 1$ ). Pero además ese flujo permanece casi constante en los tres años, si bien aquel cuyo origen está en Azerbaiyán es nítidamente superior al resto (se sitúa en torno a los 295.000 personas), mientras que cuando los emigrantes proceden de Armenia el umbral oscila alrededor de las 200.000 personas.

Tabla 1.- Origen, destino y número de refugiados.

\begin{tabular}{|l|l|r|r|r|}
\hline OFIGEN & DESTINO & \multicolumn{1}{|c|}{1992} & \multicolumn{1}{|c|}{1993} & \multicolumn{1}{c|}{1994} \\
\hline ARMENIA & AZERBAIYAN & 195000 & 229000 & 200000 \\
\hline & FED. RUSA & & 26000 & \\
\hline & & & & \\
\hline AZEREAIYAN & ARMENIA & 300000 & 290000 & 290000 \\
\hline & FED. RUSA & & 26000 & 84000 \\
\hline & & & & \\
\hline GEORGIA & FED. RUSA & & 143000 & 101000 \\
\hline & ARMENIA & & & 5800 \\
\hline & & & & \\
\hline UZBEKISTAN & AZERBAIYAN & 51000 & 51000 & 51000 \\
\hline
\end{tabular}

Fuente USCR $\left(1992,1993\right.$ y 1994) ${ }^{8}$. Elaboración propia.

8 Los refugiados de la Federación Rusa procedentes de Armenia y Azerbaiyán se han distribuido equitativamente. Es decir, se consideran 26.000 personas procedentes de cada República. Por otra parte, en Azerbaiyán se incluyen 51.000 turcos procedentes de Uzbekistán en 1989 que, si bien no son consecuencia de 
Por otro lado, los emigrantes de Armenia y Azerbaiyán que se dirigen hacia 1 a Federación Rusa difieren cuantitativamente del caso anterior, pues se trata de un flujo unidireccional y de menor entidad. En efecto, de ambas Repúblicas proceden personas en dirección a su antigua potencia protectora sin recibir población de retorno. El número de emigrantes es limitado (26.000 personas en 1993), aunque creciente desde Azerbaiyán en 1994 (84.000 personas) como consecuencia de la inestabilidad política, económica y los recientes exigencias autonomistas de lezguianos y taliches.

Los flujos de Georgia se dirigen esencialmente a la Federación Rusa. Son, por tanto, unidireccionales y decrecientes, pues si bien en 1993 se alcanzaban los 143.000 refugiados, el año siguiente se reducen a 101.000 refugiados. A estos movimientos hay que añadir los 5.800 repatriados a Armenia procedentes de Abjasia en 1994.

Todo esto se representa cartográficamente en los mapas $n^{\circ} 3,4$ y 5 , donde el grosor nos indica la intensidad y la dirección de la flecha el destino de los movimientos. En sendos mapas figura además el porcentaje de migrantes respecto a I total de población del país, siendo la República de Azerbaiyán la que alcanza los valores superiores (en torno a $13 \%$ en 1993 y 1994).

\section{DESPLAZADOS INTERNOS}

De mayor envergadura es el desplazamiento interno de personas en el ámbito de las Repúblicas caucasianas. Así, Azerbaiyán pasa de 216.000 personas en 1992 a unas 600.000 personas en los años siguientes, lo que infiere la acción de las fuerzas armenias de Nagorno-Karabaj sobre las ciudades azeríes ubicadas en las proximidades de su enclave territorial. Esto se aprecia en la Figura 6, donde los núcleos azeríes, los flujos y sus direcciones representan esos desplazamientos.

Algo similar sucede en Georgia, aunque de menor entidad, ya que si en 1992 el número de desplazados internos ascendía a 15.000 personas, en los años siguientes se alcanzan los 250.000 desplazados. La dirección, origen, y destino de esos desplazamientos están bien representados por las líneas de flujo, siendo la causa fundamental de tipo étnico con la expulsión de los georgianos residentes en esta región occidental de la República. Al mismo tiempo, en Armenia solamente se desplazan unas 75.000 personas en 1993 y 1994 debido a las acciones sobre la región de Nakitcheván, ocupada en su mayor parte por azeríes.

los conflictos de Armenia y esta República, constituyen una "carga migratoria" importante para el país receptor (USCR, 1994). 


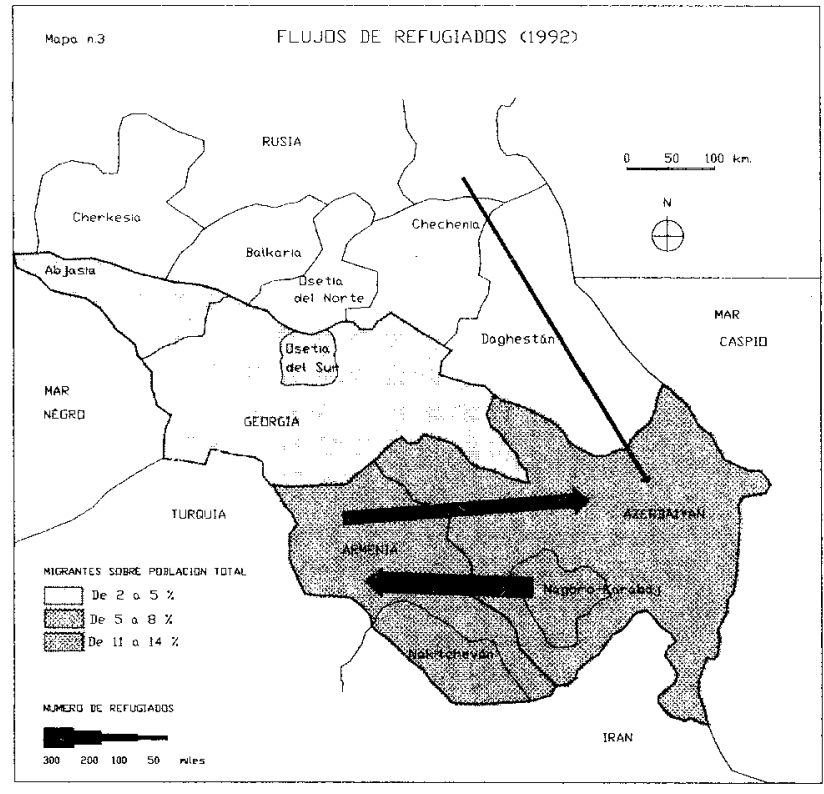

Figura 3.- Flujos de refugiados en 1992.

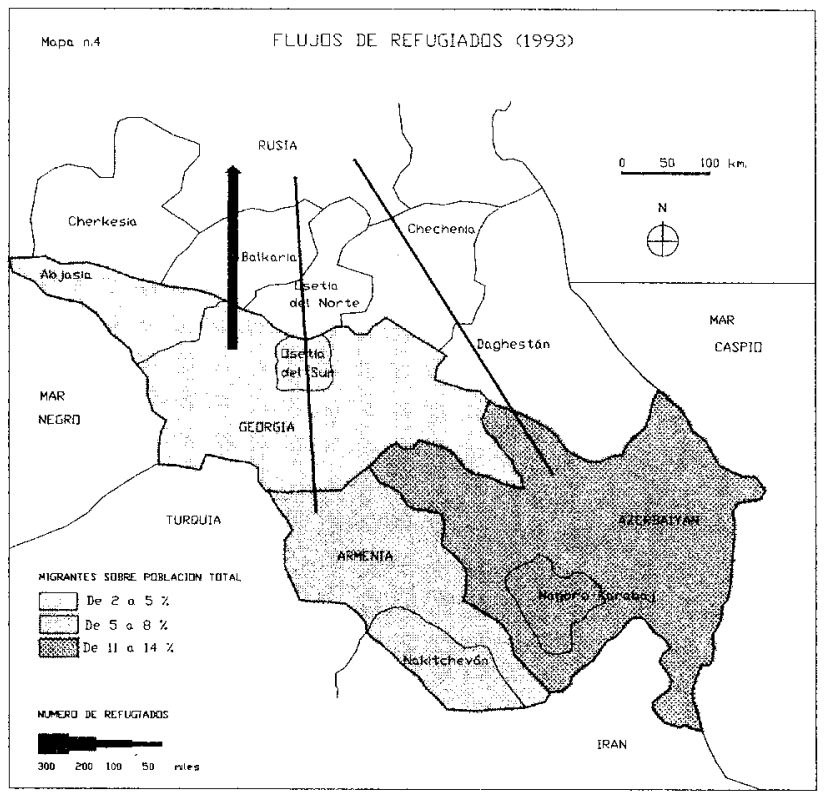

Figura 4.- Flujos de refugiados en 1993. 


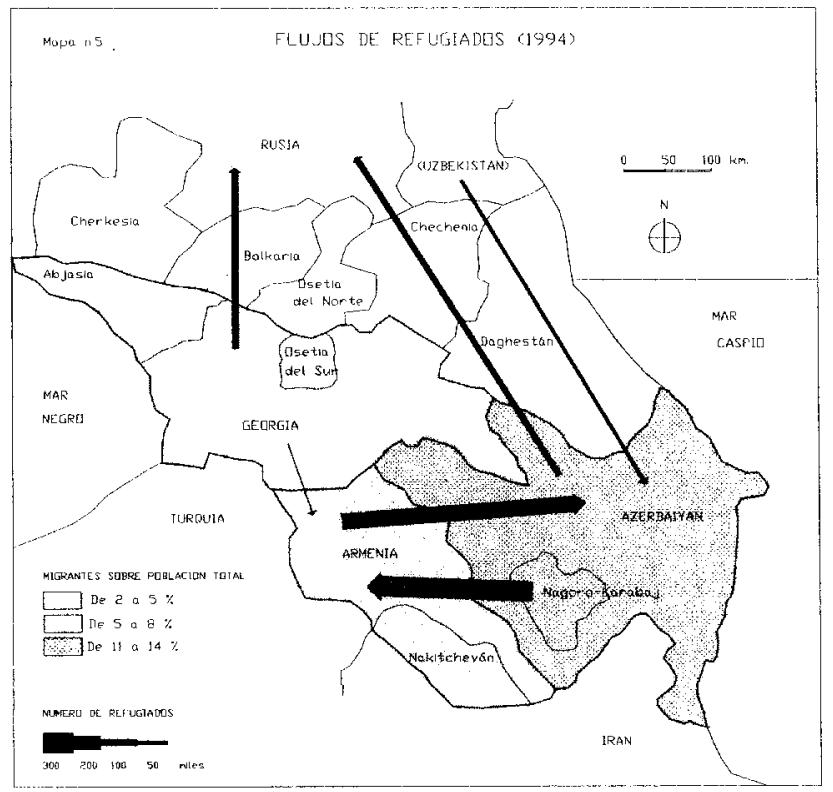

Figura 5.- Flujos de refugiados en 1994.

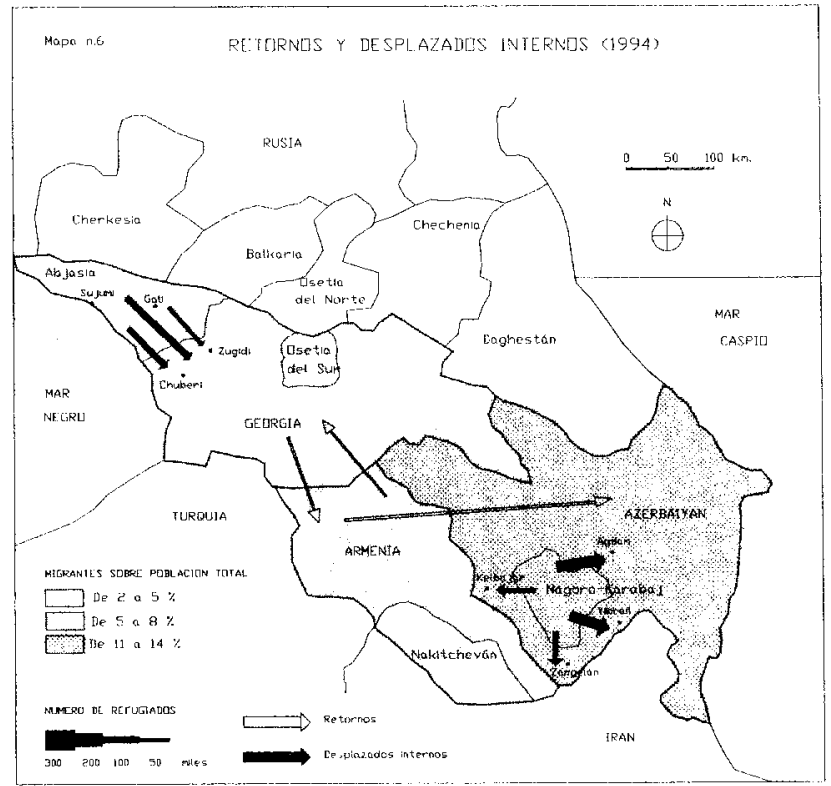

Figura 6.- Retorno y desplazados internos en 1994. 
Tabla 2.- Número de desplazados internos.

\begin{tabular}{|l|r|r|r|}
\hline & \multicolumn{1}{|c|}{1992} & \multicolumn{1}{|c|}{1993} & \multicolumn{1}{c|}{1994} \\
\hline AZERBAIYAN & 216000 & 600000 & 600000 \\
\hline ARMENIA & & 77000 & 72000 \\
\hline GEORGIA & 15000 & 250000 & 260000 \\
\hline
\end{tabular}

Fuente: USCR (1992, 1993 y 1994). Elaboración propia.

En el mismo mapa se observan los movimientos de retorno hacia los lugares de origen. Este aspecto constituye una de las principales preocupaciones de ACNUR, de tal modo que se integra como una alternativa de emigración ordenada (ACNUR, 1995). Pues bien, en el ámbito del Cáucaso sólo se han producido retornos limitados, ya que apenas alcanzan las 29.000 personas en Azerbaiyán, 5.800 en Armenia y 1.000 personas en Georgia.

\section{CONCLUSIONES}

El estudio de los movimientos migratorios en las Repúblicas del Cáucaso ha puesto de relieve la complejidad de causas y procesos decisorios que están implícitos en los desplazamientos de fuertes contingentes de población. Por su parte, el acceso a 1 a independencia de estas Repúblicas en 1991 ha propiciado el desarrollo de los recientes conflictos de secesión y anexión, aunque ciertamente en muchos casos constituyen episodios actuales de antiguas diferencias inducidas por presiones exteriores.

Los mapas de presentación de la causas fundamentales de los conflictos, de étnias implicadas y de flujos migratorios, bien de refugiados o desplazados internos, constituyen un corpus cartográfico importante que ha coadyuvado a exponer los problemas e interrelacionarlos. 
En definitiva, se han esbozado algunas características de los actuales movimientos de refugiados y desplazados en el Cáucaso, y la cartografía ha sido el medio visual que ha permitido representarlas. Este estudio es un primer paso, aunque esencial, para el conocimiento de una problemática tan relevante y grave como 1 a migración.

\section{BIBLIOGRAFIA}

ABELLÁN, A. et al. (1991): La población del mundo. Síntesis, Madrid.

ACNUR (ALTO COMISIONADO DE LAS NACIONES UNIDAS), (1994): La situación de los refugiados en el mundo. El desafio de la protección, Alianza editorial, 13-29, Madrid.

ACNUR (ALTO COMISIONADO DE LAS NACIONES UNIDAS), (1995): La situación de los refugiados en el mundo. En busca de soluciones, Alianza editorial, Madrid.

ASOCIACION CARTOGRAFICA INTERNACIONAL (1966): Comisión sobre la formación de los cartógrafos, UNESCO, París.

CALVO PALACIOS, J.L. (1992): "Concepción y ejecución de cartografía para la ordenación del territorio y el urbanismo a través de Sistemas de Información Geográfica", $V$ Coloquio de Geografía Cuantitativa, Ponencias, Institución Fernando El Católico, Zaragoza.

EL ESTADO DEL MUNDO (1993-1996): Anuario económico y geopolitico mundial, Akal, Madrid.

ESCALONA ORCAO, A. (1995): "El estudio de las migraciones de refugiados. Cuestiones teóricas y metodológicas, Geographicalia 32, 63-82, Zaragoza.

KUNZ, E.F. (1973): "The Refugee in flight: Kinetic Models and Forms of Displacement", International Migration Review, 7, pp. 125-146.

LEVINSON, D. (1993): "Conflictos étnicos y refugiados", Refugiados n”81, pp. $23-28$.

MEDICOS SIN FRONTERAS (1995): Poblaciones en peligro, Acento editorial, Madrid.

PUEYO CAMPOS, A. (1994): Utilización de cartografia para el análisis y diagnóstico de la localización de equipamientos, Universidad de Zaragoza, Zaragoza.

UNHCR (1994): Populations of concern to UNHCR. A stadistical overview, Office of the nations high commissioner for refugees.

US COMMITTEE FOR REFUGEES (1993): World Refugee Survey Washington DC: US Committees for Refugees. Comité para los Refugiados de Estados Unidos, 1993.

US COMMITTEE FOR REFUGEES (1994): World Refugee Survey Washington DC: US Committees for Refugees. Comité para los Refugiados de Estados Unidos, 1994 
US COMMITTEE FOR REFUGEES (1995): World Refugee Survey Washington DC: US Committees for Refingees. Comité para los Refugiados de Estados Unidos, 1995

WEINTRAUB, S. Y STOLP, CH. (1989): "Las consecuencias de una interdependencia económica", en Organización de Cooperación y Desarrollo Económico (OCDE), El futuro de las migraciones. Ministerio de Trabajo y Seguridad Social, colección Informes OCDE, 29, 207-254, Madrid.

WOOD, W. (1994): "Forced migration: local conflicts and international dilemmas", Annales of the Association of American Geographers, 607-634.

ZOLBERG, A. (1991): Are the industrial countries under siege? Comunicación presentada a la conferencia sobre Políticas migratorias en Europa y Estados Unidos. Roma: Centro di Studi Americani y Fondazione Enrico Mattei.

ZOLBERG, A. SUHRKE, A. \& AGUAYO, S. (1989): Escape from violence. Conflict and refugee crisis in the developing worl, Oxford University Press, 3-31, Oxford. 